Präv Gesundheitsf 2022 · 17:457-463 https://doi.org/10.1007/s11553-021-00903-3 Eingegangen: 7. Juni 2021

Angenommen: 17. August 2021

Online publiziert: 24. September 2021

c c Der/die Autor(en) 2021

Anne Ritschel · Manfred Döpfner' · Stephanie Schürmann' · Julia Plück' · Hanna Meyer' ${ }^{1}$ Marie Schottel' . Ulrike Ravens-Sieberer ${ }^{2} \cdot$ Veit Roessner $^{3}$. Tobias Banaschewski ${ }^{4} \cdot$ Anja Görtz-Dorten ${ }^{1} \cdot$ Michael Kölch $^{5,6,7} \cdot$ AnneKatrin Treier ${ }^{1}$. Charlotte Hanisch ${ }^{1}$

'Lehrstuhl für Psychologie und Psychotherapie in Heilpädagogik und Rehabilitation, Universität zu Köln, Köln, Deutschland

${ }^{2}$ Universitätsklinikum Hamburg-Eppendorf, Hamburg, Deutschland

${ }^{3}$ Technische Universität Dresden, Dresden, Deutschland

${ }^{4}$ Universität Heidelberg, Heidelberg, Deutschland

${ }^{5}$ Universität Ulm, Ulm, Deutschland

${ }^{6}$ Medizinische Hochschule Brandenburg, Neuruppin, Deutschland

${ }^{7}$ Universitätsmedizin Rostock, Rostock, Deutschland

\title{
Online-Elterntraining für die Behandlung von Kindern mit externalisierenden Verhaltensproblemen und affektiver Dysregulation
}

intensive affektive und verhaltensbezogene Reaktionen auf negative emotionale Stimuli, durch eine sog. affektive Dysregulation, auszeichnet [18]. Betroffene Kinder zeigen eine anhaltend gereizte Stimmung, affektive Labilität und intensive Wutausbrüche. Im DSM 5 werden die Symptome bei entsprechend starker Ausprägung und anhaltender Dauer mit der disruptiven Affektregulationsstörung erstmalig als eigenständige psychische Störung aufgeführt, für die eine Prävalenz von $0,8-3,3 \%$ berichtet wird [4]. Subklinisch ausgeprägte schwere Stimmungsschwankungen mit anhaltender Gereiztheit kommen allerdings deutlich häufiger vor und überschneiden sich mit externalen Störungen, Angststörungen und Depression [26]. Da affektive Labilität in der Kindheit als Prädiktor für spätere depressive Störungen und weitere psychische Störungen identifiziert wurde [29], ist die Entwicklung wirksamer Präventions- und Behandlungsmöglichkeiten von hoher klinischer Relevanz.

Ätiologische Modelle messen - neben personalen Faktoren wie Temperament und neuropsychologischen Besonderheiten - sozialen Faktoren eine besondere Bedeutung bei der Entstehung affektiver Dysregulation bei [22]. In der Interaktion mit Eltern, Gleichaltrigen und Erwachsenen außerhalb der Familie erwerben Kinder Emotionsregulationsstrategien und erproben diese Strategien im Hinblick auf die Erreichung individueller Ziele. Wenn Eltern selbst einen ungünstigen Umgang mit negativen Gefühlen aufweisen, dienen sie als Modell für das Erlernen maladaptiver Emotionsregulationsstrategien. In diesem Fall können sich aufschaukelnde dysfunktionale Interaktionsmuster zwischen Kind und Eltern entwickeln („,coercive interactions“), die mit intensiven negativen Gefühlen auf beiden Seiten einhergehen und eine hohe Stabilität aufweisen.

\section{Elterntrainings zur Behandlung von externalen Störungen}

Kognitiv-verhaltenstherapeutische Elterntrainings (sog. „parent management trainings“, PMT) gelten als evidenzbasierter Therapiebaustein für die multi- 


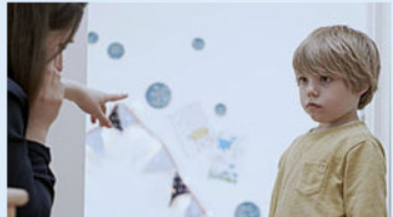

Verhaltensprobleme lösen

Hier lernen Sie wirksame Methoden für konkrete Problemsituationen mit Ihrem Kind. Kurze Videos zeigen Innen die Umsetzung der vermittelten Erziehungstipps.

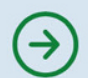

$\longrightarrow$

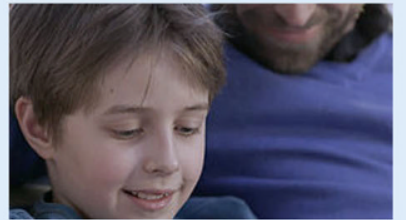

Beziehung zum Kind stärken

Hier lernen Sie, wie Sie die Beziehung zu Ihrem Kind stärken und Ihr Kind wieder mit seinen positiven Seiten wahrnehmen können.

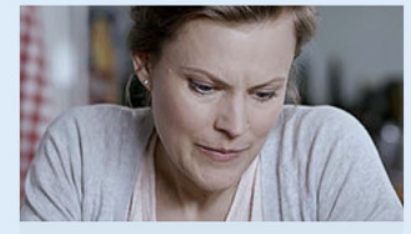

Sich selbst nicht vergessen

Wenn Sie selbst am Rande der Erschöpfung sind, können Sie hier lernen, Ihren Alltag so zu gestalten, dass weniger Stress aufkommt und feste Zeiträume für Ihre Erholung entstehen.

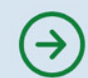

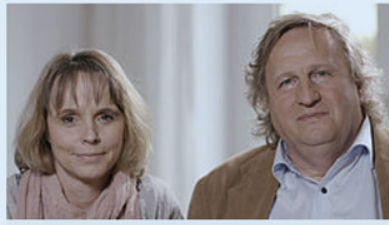

ADHS - Was ist das?

Hier erhalten Sie von Prof. Manfred Döpfner und Dr. Stephanie Schürmann Infos zu Symptomen, Ursachen und Behandlung von ADHS.
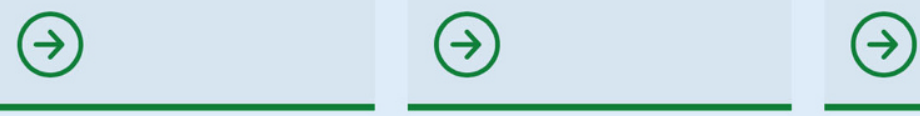

Abb. 1 \& Übersicht über die vier Trainingsbereiche des ADHS-Elterntrainers (Quelle: [31])

modale Behandlung von Kindern mit externalen Verhaltensstörungen [27]. Sie zielen daraufab, auf der Basis einer warmherzigen, wertschätzenden Eltern-KindBeziehung durch eine Veränderung von Situations- und Verstärkerbedingungen die elterliche Steuerung und Lenkung kindlichen Verhaltens zu erhöhen. In Metaanalysen werden für PMT bei externalen Auffälligkeiten mittlere Effektstärken von bis zu $d=0,68$ berichtet [3]. Im deutschen Sprachraum hat sich v. a. das Therapieprogramm für Kinder mit hyperkinetischem und oppositionellem Problemverhalten (THOP; [10]) und das davon abgeleitete Präventionsprogramm für expansives Problemverhalten (PEP; [23]) in einer Vielzahl von Studien als wirkungsvoll erwiesen. Klassische behaviorale Elterntrainings beeinflussen nur indirekt die kindliche Emotionsregulation, indem Eltern angeleitet werden, Regeln $\mathrm{zu}$ formulieren und deren Einhaltung durch positive Konsequenzen zu verstärken. Eine gezielte Verbesserung der intrapsychischen Emotionsregulation liegt dagegen nicht im Fokus dieser klassischen Elterntrainings. Für Kinder mit affektiver Dysregulation ist eine inhaltliche Erweiterung herkömmlicher kognitiv-verhaltenstherapeutischer Elterntrainings nötig, die das Kind und die Eltern zusätzlich beim Erwerb adaptiver Emotionsregulationsstrategien unterstützt. Achtsamkeitsbasierte, emotionsfokussierte Inhalte könnten hierfür eine Ergänzung darstellen [6].

\section{Der Einsatz von Selbsthilfe- programmen}

Face-to-face-Elterntrainings werden von bestimmten Elterngruppen wie z.B. alleinerziehenden, berufstätigen Elternteilen mit mehreren Kindern, weniger gut angenommen [15]. Alternativ können Selbsthilfeprogramme bei der elternzentrierten Arbeit eingesetzt werden, die sich durch geringere Behandlungskosten und -barrieren auszeichnen [21], aber auch hohe Anforderungen an die Schriftsprachkenntnisse und Selbstmanagementfähigkeiten der Eltern stellen [19]. Über angeleitete Selbsthilfeprogramme, die gedruckte Elterninformationen und Telefonberatung kombinieren, konnten in randomisierten Kontrollgruppenstudien signifikante Effekte auf externale Auffälligkeiten des Kindes, die Erziehungskompetenzen der Eltern und die elterliche Selbstwirksamkeit gemessen werden [9, 20]. Einen möglichen Mediator für den positiven Effekt dieser Programme auf kindliche Verhaltensauffälligkeiten könnte die Reduktion dysfunktionaler elterlicher Attributionen darstellen.

\section{Chancen von Online-Trainings für die Behandlung}

Eine erfolgsversprechende Behandlungsalternative können auch Online-Trainings sein, die im Wartekontrollgruppendesign ähnlich hohe Effektstärken wie Face-to-face-Trainings aufweisen [1]. Entsprechende Studien beinhalten jedoch zusätzliche Termine mit den Familien z.B. zur Datenerhebung und Verstärkung der Teilnahme, die Einfluss auf das Nutzungsverhalten nehmen können. Online-Trainings können insbesondere als erste Intervention eines gestuften Behandlungskonzepts, zur Ergänzung einer Face-to-face-Behandlung oder zur Überbrückung von Wartezeiten und Versorgungslücken genutzt werden [28]. Es gibt jedoch auch Bedingungen, die eine sofortige psychotherapeutische Behandlung im Face-to-face-Setting erfordern und für die ein vorangestelltes Online-Training nicht ausreichen würde. Dies könnte beispielsweise bei einer drohenden Chronifizierung psychischer Störungen oder einer krisenhaften $\mathrm{Zu}$ spitzung mit drohender schulischer Exklusion oder Fremdunterbringung der Fall sein. Die Adhärenz wird definiert als das Ausmaß, in dem Teilnehmende das Online-Training im vereinbarten Umfang nutzen. In einer Metaanalyse wurde für Online-Trainings mit thera- 
Präv Gesundheitsf 2022 · 17:457-463 https://doi.org/10.1007/s11553-021-00903-3

(c) Der/die Autor(en) 2021

\section{A. Ritschel · M. Döpfner · S. Schürmann · J. Plück · H. Meyer · M. Schottel · U. Ravens-Sieberer · V. Roessner · T. Banaschewski · A. Görtz-Dorten · M. Kölch · A.-K. Treier · C. Hanisch \\ Online-Elterntraining für die Behandlung von Kindern mit externalisierenden Verhaltensproblemen und affektiver Dysregulation}

\section{Zusammenfassung}

Hintergrund. Aufmerksamkeitsdefizit-/Hyperaktivitätsstörung (ADHS) oder oppositionelle Verhaltensstörung (OPP) gehen mit unruhigem, oppositionellem und aggressivem Verhalten, negativen Interaktionen zwischen Kind und Eltern und langfristig häufig mit belasteten Eltern-Kind-Beziehungen einher. Kognitiv-behaviorale Elterntrainings, die für die Behandlung externaler Störungen als evidenzbasiert gelten, versuchen problematische situative und Verstärkerbedingungen sowie negative Interaktionen zwischen Eltern und Kindern zu verändern.

Ziel. Es sollen Online-Selbsthilfetrainings für Eltern von Kindern mit externalisierenden Verhaltensproblemen entwickelt werden. Methode. Bestehende Face-to-faceElterntrainings wurden in Online-Formate überführt und inhaltlich erweitert. Eine Wirksamkeitsuntersuchung erfolgt im Rahmen von randomisierten Kontrollstudien. Ergebnisse. Der ADHS-Elterntrainer richtet sich an Eltern von Kindern mit subklinischen sowie klinisch relevanten ADHS-Symptomen sowie ggf. komorbid weiteren externalen Symptomen. Er zielt darauf ab, Störungsverständnis aufzubauen, die elterliche Steuerung zu erhöhen und die Eltern-KindBeziehung zu verbessern. Bei Kindern mit Emotionsregulationsstörungen, sog. affektiver Dysregulation, ist darüber hinaus die elterliche Unterstützung beim Erwerb funktionaler Emotionsregulationsstrategien nötig. Für das ADOPT-Online-Elterntraining (affektive Dysregulation - Optimierung von Prävention und Therapie), das sich an Eltern von Kindern mit affektiver Dysregulation richtet, wurde das Konzept des ADHS-Elterntrainers um Interventionen zum Umgang mit negativen Gefühlen des Kindes und der Eltern erweitert. Diskussion. Bei entsprechend positiven Effekten auf die kindliche Symptomatik innerhalb der Evaluationsstudien könnte das ADOPT-Online-Elterntraining wie bereits beim ADHS-Elterntrainer geschehen im präventiven oder therapeutischen Setting eingesetzt werden.

\section{Schlüsselwörter}

Externale Störungen · ADHS · Oppositionelle Verhaltensstörung · Emotionsregulation . Kognitiv-behaviorale Therapie

\section{Online parent training for the treatment of children with externalizing behavioral problems and affective dysregulation}

\section{Abstract}

Background. Externalizing disorders, including attention deficit hyperactivity disorder (ADHD) and oppositional defiant disorder (ODD), often lead to persistent maladaptive patterns in the interaction between children and their parents. These patterns are characterized by dysfunctional antecedents and consequences and by aggressive behavior and can lead to negative parent-child relationships.

Objective. The aim is to develop online training in a self-help concept that targets parents of children with externalizing disorders.

Methods. Existing face to face parent training has been transferred into online formats and the content enhanced. The effectiveness is examined in randomized controlled trials. Results. The ADHD parent trainer addresses parents of children with subclinical or clinically relevant ADHD and other coexisting external symptoms. It aims at improving knowledge about the disorder, strengthening the relationship between parent and child, and improving parental control. Affective dysregulation summarizes symptoms of irritation, affective lability and excessive anger and aggression. In order to reduce problem behavior, children with affective dysregulation first need to learn adaptive strategies for the management of unpleasant feelings. For the Affective Dysregulation -Optimizing Prevention and Therapy (ADOPT) Online parent training, which addresses parents of children with affective dysregulation, the ADHD parent trainer was expanded by interventions that focus on an adaptive management of unpleasant feelings. Discussion. If the running evaluation study reveals positive effects on child symptoms, the ADOPT Online parent training could be implemented for preventive or therapeutic settings as has already been the case for the ADHD parent trainer.

\section{Keywords}

Externalizing disorders · ADHD · Oppositional defiant disorder - Emotion regulation . Cognitive-behavioral therapy peutischem Kontakt eine durchschnittliche Adhärenz von $50 \%$ ermittelt [16]. Einzelne nicht-angeleitete Online-Elterntrainings berichten für 7,5-22,8\% der Teilnehmenden eine vollständige Nutzung [5, 7]. Es finden sich Hinweise darauf, dass sich spielerische technische Features („gamification“) förderlich auf die Adhärenz auswirken [13].

\section{Die Konzeption von Online- Elterntrainings für die Behandlung von Kindern mit externalen Störungen}

Forschungsgruppen an der Universitätsklinik Köln und der Universität zu Köln haben Online-Trainings entwickelt, die Eltern von Kindern mit externalen Störungen unterstützen sollen. Die Wirk- samkeit dieser Interventionen wird aktuell über randomisierte Kontrollgruppenstudien untersucht $[8,12]$.

\section{Der ADHS-Elterntrainer}

In Kooperation mit dem AOK-Bundesverband wurde auf der Grundlage des Therapieprogramms für Kinder mit hyperkinetischem und oppositionellem 


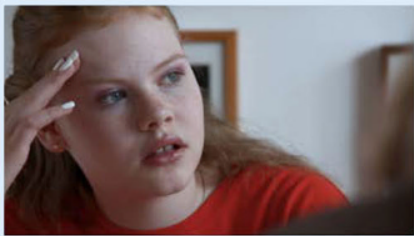

Ihr Kind stärken

Hier erfahren Sie, wie Sie Ihr Kind im Umgang mit schwierigen Gefühlen unterstützen können.

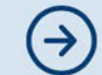

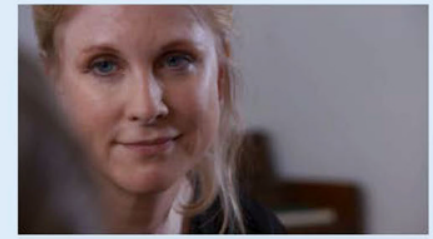

Sich selbst stärken

Hier können Sie selbst einen guten Umgang mit schwierigen Gefühlen erlernen.

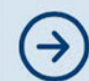

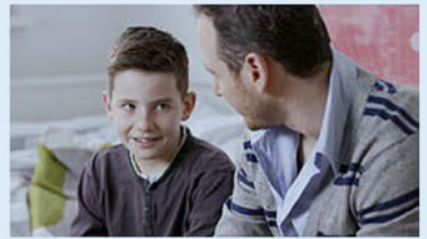

Gefühle: Wissenswertes

Hier finden Sie alles Wissenswerte zu Problemen im Umgang mit schwierigen Gefühlen.

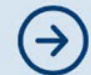

Abb. $2 \triangleleft$ Übersicht über die drei Trainingsbereiche des ADOPT-Online-Elterntrainings
Problemverhalten (THOP) und eines davon abgeleiteten Selbsthilfebuches [11] ein Online-Selbsthilfeformat für Eltern von Kindern mit ADHS und oppositionellen Verhaltensstörungen entwickelt, das Eltern anonym und kostenlos nutzen können [25] (https://adhs.aok.de/zumadhs-elterntrainer/). Im Online-Training erwerben Eltern Wissen über ADHS und lernen verhaltenstherapeutisch basierte Strategien, um Verhaltensprobleme ihres Kindes in der Familie zu reduzieren, die Eltern-Kind-Beziehung zu stärken und eigene Ressourcen zu aktivieren (• Abb. 1).

Im Trainingsbereich „ADHS - Was ist das?" werden den Eltern wissenschaftlich fundierte Informationen zu ADHS vermittelt, die dysfunktionale Einstellungen dem Kind gegenüber verändern sollen. Im Trainingsbereich „Sich selbst nicht vergessen“ reflektieren die Eltern Belastungen und werden dazu angeregt, Ressourcen $\mathrm{zu}$ aktivieren und hilfreiche Strukturen im Alltag über Routinen und Wochenpläne $\mathrm{zu}$ etablieren. Im Trainingsbereich „Beziehung zum Kind stärken“ werden die Eltern dazu angeleitet, sich auf positive Eigenschaften des Kindes zu fokussieren, gemeinsam positiven Aktivitäten nachzugehen und das Kind über eine positivere soziale Integration und Erfolge zu stärken. Der größte Trainingsbereich „Verhaltensprobleme lösen“ setzt gezielt an Problemsituationen an, die von Eltern mit Kindern, die externale Verhaltensprobleme aufweisen, häufig als herausfordernd erlebt werden (Hausaufgaben, Medienzeit, Kinderzimmer aufräumen, Unruhe beim Essen, Ständiges Unterbrechen, Geschwisterstreit, Wutausbrüche). Anhand des nach persönlicher Relevanz frei wählbaren Problembereichs lernen Eltern videogeleitet sieben Schritte zur Problemlösung kennen: Problemanalyse, Identifikation des interaktionellen Teufelskreises, Aufstellen von Regeln, wirkungsvolle Aufforderungen, natürliche positive und negative Konsequenzen und Belohnungspläne. Dieses systematische Vorgehen kann für alle Problemsituationen durchlaufen werden.

\section{Das ADOPT-Online-Eltern- training}

Das ADOPT-Online-Elterntraining wurde als Teilprojekt „ADOPT Online“ des Verbundprojekts ADOPT entwickelt und richtet sich als erste Maßnahme einer gestuften Intervention an Eltern von Kindern mit affektiver Dysregulation [8]. Es erweitert die Inhalte des ADHS-Elterntrainers um weitere, mit denen Eltern die kindliche und auch ihre eigene Emotionsregulation verbessern sollen (• Abb. 2).

Grawes Grundbedürfnismodell [14], welches eine möglichst ausgewogene Befriedigung der kindlichen Bedürfnisse nach Orientierung/Kontrolle, Bindung/Zugehörigkeit, Selbstwerterhöhung/-schutz und Lustgewinn/
Unlustvermeidung vorsieht, dient hierbei als theoretische Grundlage. Kinder mit affektiver Dysregulation benötigen bei Eingrenzung, Anforderung oder Misserfolg Unterstützung im Umgang mit negativen Emotionen, um sich letztendlich als selbstwirksam und erfolgreich erleben zu können. Eine Befriedigung der Bedürfnisse nach Orientierung, Anerkennung und Selbstwirksamkeit setzt oftmals eine erfolgreiche Emotionsregulation voraus. Im Sinne einer Integration von Methoden der dritten, sogenannten „emotionalen Wende“ der Verhaltenstherapie in das Elterntraining sollen die Eltern zu einer achtsamen, d.h. aufmerksamen und akzeptierenden Haltung gegenüber Gefühlen angeregt werden.

Das ADOPT-Online-Elterntraining versucht, Eltern dieses Zusammenspiel über das Bild eines „dreibeinigen Hockers“ zu vermitteln: Damit das Kind stabil auf dem „Entwicklungs-Hocker“ sitzen kann, braucht dieser drei „gleichlange Beine": Das Kind braucht (1) Unterstützung beim Umgang mit negativen Gefühlen, um sich als selbstwirksam und erfolgreich erleben zu können, erfährt durch (2) klare Strukturen und elterliche Lenkung Orientierung und fühlt sich (3) auf der Basis warmherziger Zuwendung sicher gebunden und unterstützt (- Abb. 3).

Angelehnt an das Konzept des „mindful parenting“ [2] werden die Eltern im ADOPT-Online-Elterntraining in einer 


\section{Willkommen liebe Eltern!}

Schön, dass Sie da sind und Ihr Kind im Umgang mit seinen Gefühlen unterstützen wollen. Damit tun Sie Ihrem Kind einen großen Gefallen, denn Kinder mit Problemen im Umgang mit schwierigen Gefühlen haben häufig Konflikte mit Eltern, Freunden oder in der Schule. Das kann Ihr Kind verunsichern und traurig machen.

Stellen Sie sich Ihre Erziehung wie einen Hocker mit 3 Beinen vor. Damit sich Ihr Kind gesund entwickeln kann und nicht wackelt, braucht es Unterstützung im Umgang mit Gefühlen, damit es gut im Leben zurechtkommt. Es braucht Struktur durch klare Regeln und Lob, damit es Orientierung in der Welt findet. Und es braucht Zuwendung, damit es sich geliebt fühlt.

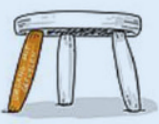

Umgang mit Gefühlen

Helfen Sie Ihrem Kind mit dem 5-Schritte-Plan, mit seinen schwierigen Gefühlen umzugehen.

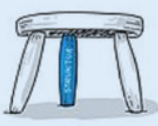

\section{Struktur}

Lernen Sie Regeln so aufzustellen, dass sich Ihr Kind daran hält und strukturieren Sie Ihren Alltag klar.

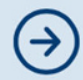

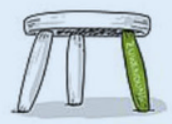

Zuneigung

Vermitteln Sie Ihrem Kind die Zuneigung, die es braucht.

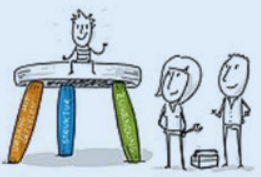

\section{Alle 3 Beine zusammen}

Sehen Sie sich anhand von kurzen Spielfilmen an, wie man den Umgang mit Gefühlen, Struktur und Zuneigung verbinden kann.
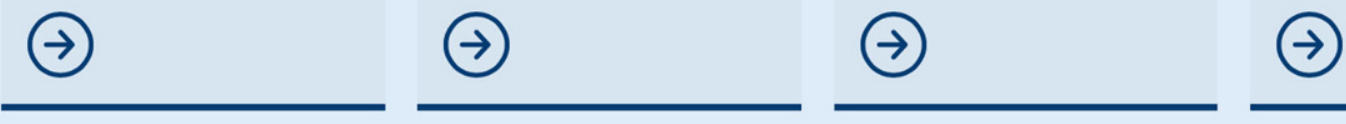

Abb. $3 \Delta$ Modularer Aufbau des ADOPT-Online-Elterntrainings: Ihr Kind stärken

achtsamen Wahrnehmung eigener und kindlicher Gefühle unterstützt. Sie werden so zum einen angeleitet, eigene Reaktionsmuster früher zu erkennen und sich zum anderen in die kindliche Perspektivehineinzuversetzen. Hierfür können die Eltern Meditationsübungen nutzen oder ihr Kind achtsam in Alltagssituationen beobachten. Eltern von Kindern mit affektiver Dysregulation erleben aufgrund ungünstiger Emotionsregulationen und Erfahrungen von schwierigen Interaktionen mit hoher Wahrscheinlichkeit selbst mehr negative Emotionen. Daher können die Eltern im ADOPT-Online-Elterntraining auch Strategien erlernen, mit denen sie sich selbst stärken. Neben dem Kind- und Elternmodul gibt es ein Edukationsmodul (• Abb. 2).

Zentrale Intervention zur Verbesserung der Emotionsregulation ist der sog. ,5-Schritte-Plan“ (Schritt 1: Stopp!, Schritt 2: Was fühle ich?, Schritt 3: Ich gehe liebevoll mit mir um., Schritt 4: Ich denke etwas Hilfreiches, Schritt 5: Ich tue etwas Hilfreiches). Er kombiniert eine achtsame Wahrnehmung eigener Gefühle mit einer selbstmitfühlenden, akzeptierenden Haltung und der Anwendung kognitiver und/oder verhaltensbezogener Emotionsregulationsstrategien ([30]; - Abb. 3).

\section{Umsetzung und Evaluation}

Beide Elterntrainings können über PC, Tablet und Smartphone genutzt werden. Um die Motivation und Compliance der Eltern zu stärken, sind die Elterntrainer mit verschiedenen didaktischen Elementen ausgestattet: Über Videos, Text-Multis (Ausfüllfelder zur Beantwortung offener Fragen), Slider, Checklisten, Kalender und Erinnerungs-E-Mails sollen die Eltern dazu angeregt werden, das Training aktiv zu nutzen und den Alltagstransfer zu schaffen. Die modellhafte Aneignung der Inhalte wird dadurch unterstützt, dass die Eltern in den Modulen und Bereichen immer wieder auf die gleichen Beispielfamilien treffen. Inhalte werden über Bilder, Audios und Vi- deos (sowohl gezeichnete Erklärvideos als auch Videos mit Schauspieler*innen) veranschaulicht. Um zur Selbstreflexion anzuregen, werden die Eltern an verschiedenen Stellen aufgefordert, ihre eigene Situation in Bezug auf den spezifischen Trainingsinhalt einzuschätzen. Darüber hinaus werden Übungen vorgeschlagen, welche im Trainer protokolliert und in einem privaten Bereich gespeichert werden können. Knappe Zusammenfassungen der einzelnen Themenbereiche können als Memokarten ausgedruckt werden.

Der ADHS-Elterntrainer steht Familien bereits online zur Verfügung. Falls die laufende ADOPT-Studie eine Wirksamkeit des ADOPT-Online-Elterntrainings belegen sollte, könnte auch hier eine Implementierung in die Routinebehandlung realisiert werden. OnlineTrainings können hierbei als präventives Angebot, als niedrigschwelliges Selbsthilfeinstrument zur Überbrückung von Wartezeiten oder in Kombination mit einer Face-to-face-Behandlung eingesetzt 
werden [28]. Gleichzeitig stellen OnlineTrainings hohe Ansprüche an Familien hinsichtlich personaler Ressourcen (Sprache, Selbstmanagement) und sozioökonomischer Strukturen. Familien, bei denen die eigenständige Aneignung von Inhalten eine große Hürde darstellt, könnten über ein ergänzendes telefonisches Beratungsangebot parallel zum Online-Selbsthilfetraining erreicht werden [9].

\section{Fazit für die Praxis}

\section{- Für die Behandlung von externalen Störungen bei Kindern sind „parent management trainings" ein evidenz- basierter Behandlungsbaustein. \\ - Bei affektiver Dysregulation benö- tigen betroffene Kinder und ihre Eltern zusätzlich Interventionen zur funktionalen Emotionsregulation. \\ - Online-Selbsthilfetrainings wie der ADHS-Elterntrainer (https://adhs. aok.de) können präventiv im Selbst- hilfeformat und im Rahmen einer Psychotherapie eingesetzt werden.}

\section{Korrespondenzadresse}

Anne Ritschel
Lehrstuhl für Psychologie
und Psychotherapie
in Heilpädagogik und
Rehabilitation, Universität zu
Köln
Klosterstr. 79a, 50931 Köln,
Deutschland
anne.ritschel@uni-koeln.de

Förderung. Die diesem Beitrag zugrundeliegenden Studien wurden mit Mitteln des Bundesministeriums für Bildung und Forschung (Förderkennzeichen 01GL1741D) gefördert.

Funding. Open Access funding enabled and organized by Projekt DEAL.

\section{Einhaltung ethischer Richtlinien}

Interessenkonflikt. M. Döpfner, A. Görtz-Dorten, S. Schürmann und J. Plück erhalten Honorare für die Autorenschaft an verschiedenen Büchern, Diagnostik- und Therapiemanualen über Verhaltenstherapie bei Kindern und Jugendlichen. Dies betrifft auch die Behandlungsmanuale, die im Rahmen dieser Studie evaluiert werden. C. Hanisch erhält als Autorin eines Therapiemanuals Honorare vom Verlag. M. Kölch erhält Honorare für die Autorenschaft an verschie- denen Büchern. Er ist als Hauptprüfer (PI oder $\mathrm{Cl}$ ) in klinischen Studien für Lundbeck, Pascoe und Janssen-Cilag tätig. Außerdem erhält er Studienförderung vom BMBF, BMFSFJ sowie der BZgA und Bundeswehr. Die vorliegende Arbeit steht in keiner Verbindung zu den genannten Förderungen. T. Banaschewski ist in beratender Funktion für Lundbeck, Medice, Neurim Pharmaceuticals, Oberberg GmbH und Shire tätig. Er hat Unterstützung für Konferenzen oder Referentenhonorare von Lilly, Medice, Novartis und Shire erhalten. Außerdem war er in klinische Studien einbezogen, die von Shire und Vifor Pharma durchgeführt wurden. Er erhält Autorenhonorare von Hogrefe, Kohlhammer, CIP Medien und Oxford University Press. Die vorliegende Arbeit steht in keiner Verbindung zu den genannten Förderungen. A. Ritschel, H. Meyer, M. Schottel, U. Ravens-Sieberer, V. Roessner und A.-K. Treier geben an, dass kein Interessenkonflikt besteht.

Alle beschriebenen Untersuchungen am Menschen wurden mit Zustimmung der zuständigen Ethik-Kommissionen, im Einklang mit nationalem Recht sowie gemäß der Deklaration von Helsinki von 1975 (in der aktuellen, überarbeiteten Fassung) durchgeführt. Von allen beteiligten Patient:innen liegt eine Einverständniserklärung vor.

Open Access. Dieser Artikel wird unter der Creative Commons Namensnennung 4.0 International Lizenz veröffentlicht, welche die Nutzung, Vervielfältigung, Bearbeitung, Verbreitung und Wiedergabe in jeglichem Medium und Format erlaubt, sofern Sie den/die ursprünglichen Autor(en) und die Quelle ordnungsgemäßnennen, einen Link zur Creative Commons Lizenz beifügen und angeben, ob Änderungen vorgenommen wurden.

Die in diesem Artikel enthaltenen Bilder und sonstiges Drittmaterial unterliegen ebenfalls der genannten Creative Commons Lizenz, sofern sich aus der Abbildungslegende nichts anderes ergibt. Sofern das betreffende Material nicht unter der genannten Creative Commons Lizenz steht und die betreffende Handlung nicht nach gesetzlichen Vorschriften erlaubt ist, ist für die oben aufgeführten Weiterverwendungen des Materials die Einwilligung des jeweiligen Rechteinhabers einzuholen.

Weitere Details zur Lizenz entnehmen Sie bitte der Lizenzinformation auf http://creativecommons.org/ licenses/by/4.0/deed.de.

\section{Literatur}

1. Bennett SD, Cuijpers $P$, Ebert DD, McKenzie Smith M, Coughtrey AE, Heyman I, Manzotti G, Shafran R(2019) Practitioner review: unguided and guided self-help interventions for common mental health disorders in children and adolescents: a systematic review and meta-analysis. J Child Psychol Psychiatry 60(8):828-847

2. Bögels S, Restifo K (2014) Mindful Parenting - Achtsamkeit und Selbstfürsorge für Eltern Das Manual für ein 8-Wochen-Programm. Arbor, Freiburg

3. Coates J, Taylor JA, Sayal K (2015) Parenting interventions for ADHD: A systematic literature review and meta-analysis. J Atten Disord 19:831-838

4. Copeland WE, Angold A, CostelloEJ, Egger H (2013) Prevalence, comorbidity, and correlates of DSM-5 proposed disruptive mood dysregulation disorder. Am J Psychiatry 170(2):173-179
5. Dadds MR, Sicouri G, Piotrowska PJ, Collins DAJ, Hawes DJ, Moul C, Lenroot RK, Frick PJ, Anderson V, Kimonis ER, Tully LA (2018) Keeping parents involved: Predicting attrition in a self-directed, online program for childhood conduct problems. J Clin Child Adolesc. https://doi.org/10.1080/ 15374416.2018.1485109

6. David OA, David D, Dobrean A (2014) Efficacy of the Rational Positive Parenting Program for child externalizing behavior: can an emotion-regulation enhanced cognitive-behavioral parent program be more effective than a standard one? JEvid Based Pychother 14(2):159-178

7. Day JJ, Sanders MR (2018) Do parents benefit from help when completing a self-guided parenting program online? A randomized controlled trial comparing Triple $\mathrm{P}$ Online with and without telephone support. Behav Ther 49:1020-1038

8. Döpfner M, Katzmann J, Hanisch C, Fegert JM, Kölch M, Ritschel A, Treier AK, Hellmich M, Roessner V, Ravens-Sieberer U, Banaschewski T, GörtzDorten A (2019) Affective dysregulation in childhood-Optimizing prevention and treatment: Protocol of three randomized controlled trials in the ADOPT study. BMC Psychiatry. https://doi.org/ 10.1186/s12888-019-2239-8

9. Döpfner M, Liebermann-Jordanidis $H$, Kinnen $C$, Hallberg N, Mokros L, Benien N, Mütsch A, Schürmann S, Metternich-Kaizman WT, Hautmann C, Dose C (2018) Long-term effectiveness of guided self-help for parents of children with ADHD in routine care-An observational study. J Atten Disord. https://doi.org/10.1177/1087054718810797

10. Döpfner M, Schürmann S, Frölich J (2019) Therapieprogramm für Kinder mit hyperkinetischem und oppositionellem Problemverhalten (THOP), 6. Aufl. Beltz, Weinheim

11. Döpfner M, Schürmann S (2017) Wackelpeter und Trotzkopf-Hilfen fürEltern bei ADHS-Symptomen, hyperkinetischem und oppositionellem Verhalten, 5. Aufl. Beltz, Weinheim

12. Döpfner M, Wähnke L, Klemp MT, Mühlenmeister J, Schürmann S, Hellmich M, Plück J (2020) Efficacy of web-assisted self-help for parents of children with ADHD (WASH) - A three-arm randomized trial under field/ routine care conditions in Germany. BMC Psychiatry 20:76

13. Fleming TM, Beurs D, Khazaal $Y$, Gaggioli $A$, Rive G, Botella C, Banos RM, Aschieri F, Bavin LM, Kleinboer A, Merry S, Lau HM, Riper H (2016) Maximizing the impact of e-therapy and serious gaming: Time for a paradigm shift. Front Psychiatry $7(65): 1-7$

14. Grawe K (2000) Psychologische Therapie. Hogrefe, Göttingen

15. Heinrichs N, Bertram H, Kuschel A, Hahlweg K (2005) Parent recruitment and retention in a universal prevention program for child behavior and emotional problems: Barriers to research and program participation. Prev Sci 6(4):75-286

16. Kelders SM, Kok RN, Ossebaard HC, van GemertPijnen JE (2012) Persuasive system design does matter: a systematic review of adherence to webbased interventions. J Med Internet Res 14(6):e152

17. Larson K, Russ SA, Kahn RS, Halfon N (2011) Patterns of comorbidity, functioning and service use for US children with ADHD. Pediatrics 127(3):462-470

18. Leibenluft $E$, Stoddard J (2013) The developmental psychopathology of irritability. Dev Psychopathol 25:1473-1487

19. Lundahl B, Risser HJ, Lovejoy MC (2006) A metaanalysis of parent training: moderators and followup effects. Clin Psychol Rev 26(1):86-104 
20. McGrath PJ, Lingley-Pottie $P$, Thurston $C$, MacLean C, Cunningham C, Waschbusch DA, Watters C, Stewart S, Bagnell A, Santor D, Chaplin W (2011) Telephone-based mental health interventions for child disruptive behavior or anxiety disorders: Randomized trials and overall analysis. J Am Acad Child Adolesc Psychiatry 50:1162-1172

21. Mokros L, Benien N, Mütsch $A$, Kinnen $C$, Schürmann $S$, Metternich-Kaizman WT, Breuer D, Hautmann C, Ravens-Sieberer U, Klasen F, Döpfner M (2015) Angeleitete Selbsthilfe für Eltern von Kindern mit Aufmerksamkeitsdefizit-/ Hyperaktivitätsstörung. Konzept, Inanspruchnahme und Effekte eines bundesweiten Angebotes Eine Beobachtungsstudie. Z Kinder Jugendpsychiatr Psychother 43(4):275-288

22. Moore AA, Lapato DM, Brotman MA, Leibenluft E, Aggen SH, Hettema JM, Silberg JL, RobersonNay R (2019) Heritability, stability, and prevalence of tonic and phasic irritability as indicators of disruptive mood dysregulation disorder. J Child Psychol Psychiatry. https://doi.org/10.1111/jcpp. 13062

23. Plück J, Wieczorrek E, Metternich WT, Döpfner M (2006) Präventionsprogramm für Expansives Problemverhalten (PEP) - Ein Manual für Elternund Erziehergruppen. Hogrefe, Göttingen

24. Polanczyk GV, Salum GA, Sugaya LS, Caye A, Rohde LA (2015) Annual research review: A metaanalysis of the worldwide prevalence of mental disorders in children and adolescents. J Child Psychol Psychiatry 56(3):345-365

25. Schürmann S, Döpfner M (2018) ADHS-Elterntrainer - Ein Onlinetraining zur Selbsthilfe für Eltern von Kindern mit ADHS und oppositionellem Verhalten. Pädiatr Prax 90:1-10

26. Shaw P, Stringaris A, Nigg J, Leibenluft E (2014) Emotional dysregulation in attention deficit hyperactivity disorder. Am J Psychiatry 171:276-293

27. Sukhodolsky DG, Smith SD, McCauley SA, Ibrahim K, Piasecka JB (2016) Behavioral interventions for anger, irritability, and aggression in children and adolescents.J Child Adolesc Psychopharmacol 26:58-64

28. Tutus D, Plener PL, Niemitz M (2018) Qualitätskriterien internetbasierter kognitiv-behavioraler Interventionen für Kinder und Jugendliche sowie deren Eltern - Ein systematisches Review.Z Kinder Jugendpsychiatr Psychother 48(1):1-17

29. Vidal-Ribas P, Brotman MA, Salum GA, Kaiser A, Meffert L, Pine DS, Leibenluft E, Stringaris A (2018) Deficits in emotion recognition are associated with depressive symptoms in youth with disruptive mood dysregulation disorder. Depress Anxiety 35(12):1207-1217

30. Webb TL, Miles E, Sheeran P (2012) Dealing with feeling - A meta-analysis of the effectiveness of strategies derived from the process model of emotion regulation. Psychol Bull 138(4):775-808

31. Döpfner M, Schürmann S (2016) ADHS-Elterntrainer. Verfügbar unter: www.adhs-elterntrainer.de. Zugegriffen:28.04.2020 